\title{
Advances in Biomarkers for Lung Disease
}

\author{
Evan Kimber \\ Editorial Assistant \\ Citation: EMJ Respir. 2021;9[1]:26-27.
}

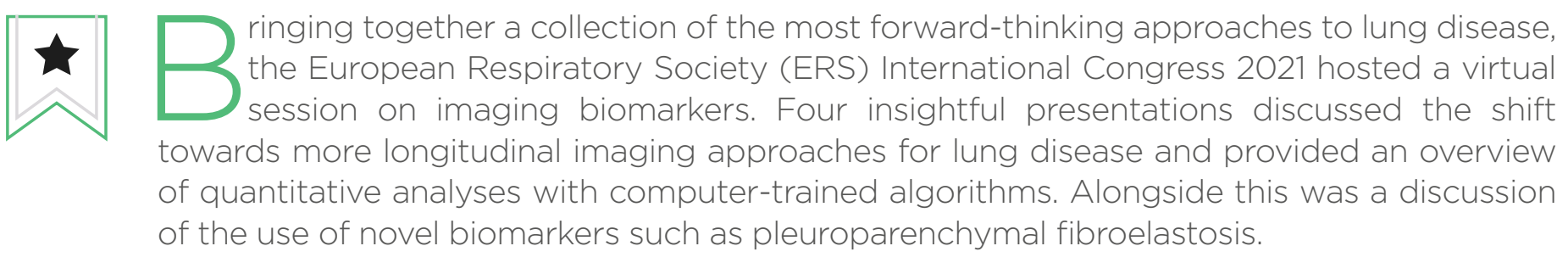

\section{QUANTITATIVE TECHNIQUES}

Opening the session, James Eaden, University of Sheffield, Sheffield Teaching Hospitals, UK, began by delivering a cross-sectional summary of the current options for quantitative imaging of lung disease. Eaden emphasised the usefulness of quantitative CT analysis for diagnostic, predictive, and prognostic purposes, enabling clinicians to objectively measure disease progression on consecutive scans. Focusing on the automated texture analysis techniques, which can be applied to the study of interstitial lung disease (ILD), Eaden highlighted that some specific automated texture analysis techniques can identify features on CT that are not visually detectable by humans.

Eaden went on to contextualise several clinical case reports of MRI with difficult ILD, cystic fibrosis, and chronic obstructive pulmonary disease. According to Eden, this field could contribute to providing diagnostic support to centres lacking expertise in thoracic imaging by stratifying patients in clinical studies. He went on to mention barriers to the research environment and limits to the software at present, including the lack of commercially availabile analysis software and the requirement for significant computational power to run analyses.

In his summary of a number of methods,
Eaden ran through several computer-aided techniques, which were later referenced by Joseph Jacob of University College London, UK. Jacob described Computer Aided Lung Informatics for Pathology Evaluation and Rating (CALIPER) variable analysis and pleuroparenchymal fibroelastosis in idiopathic pulmonary fibrosis (IPF). In line with Eaden's presentation, Jacob emphasised that computer scores are more sensitive than visual analysis. Adaptive multiple features method, quantitative lung fibrosis score, and functional respiratory imaging were among the methods discussed in the context of deep learning methods being used to train algorithms to classify high-resolution CT scans.

\section{NOVEL IMAGING BIOMARKERS}

The main body of the session discussed new approaches to using biomarkers for the assessment of lung disease. Eaden outlined hyperpolarised 129-xenon MRI as a quantitative biomarker of gas exchange, which is useful in producing ratio maps with a higher sensitivity to disease progression than pulmonary function tests for IPF and other fibrotic ILD subtypes. Building on this, the next speaker, Irma Mahmutovic Persson, Lund University, Sweden, reviewed recent preclinical and translational 
studies by describing new biomarkers identified by imaging methods. Persson stated succinctly: "The way forward in the development of biomarkers for diagnosis, but also for follow-up monitoring of lung disease, is using imaging biomarkers."

Persson explained that this pathway will involve lung injury models and translational studies with imaging, to bridge the gap and allow crossover between preclinical and clinical studies. The speaker outlined the bleomycin model in the new perspective of implementing multimodality imaging, and also referred to preclinical imaging systems, including combining $\mathrm{MRI}$ and CT/PET. This multimodal approach tackles the difficulties in imaging the lungs caused by internal organ 'noise'. In her concluding remarks, Persson described lung injury models using a systematic review, which she co-authored. The review assessed lung disease and what type of pathology the respective models mimicked, and it was narrowed from over 5,000 to 182 studies, including exclusively live imaging models.

Other speakers also discussed novel imaging biomarkers; during her talk, Greetje Vande Velde, of the $\mathrm{KU}$ Leuven in Belgium, explained that micro-CT can be useful for producing 3D visualisation based on X-rays taken from different angles. This non-invasive and dynamic technique allows effective evaluation of disease progression, and can be performed both longitudinally and quantitatively. Vande Velde mentioned that micro-CT implements simple biomarkers to find total lung volume and density from the interaction of aerated and non-aerated lung volume. In his concluding remarks on biomarkers, Jacob discussed CALIPER variable analysis in IPF. Findings show the vessels are destroyed in areas of fibrosis, and blood is diverted to lower pressure areas to allow gas exchange to take place. This means that the vessel-related structures are subsequently enlarged and more are quantified by the computer, acting as a surrogate marker of the extent of ILD. In using pleuroparenchymal fibroelastosis to identify subtypes of IPF, Jacob remarked that there are increased areas of triangular opacity in patients, making PPFE a suitable biomarker for independently predicting both forced vital capacity decline and mortality in IPF.

\section{LONGITUDINAL ANALYSES}

A common theme throughout the session was the requirement for longitudinal design intervention. Jacob mentioned that, currently, challenges lie with the data and the low amount of longitudinal imaging. This makes a case for the requirement for standardised imaging practice. Jacob also outlined the existing issue with outdated radiological terminology, where scanning practice has improved but is limited by 20-year-old vocabulary. Jacob suggested we are blind to the potential new imaging features that come with a long-term approach, and described an inability to identify these features in current practice.

"I am a very big fan of longitudinal imaging and I think that it is the key if you are going to track disease progression," was Persson's clear supporting statement for longitudinal analyses. Vande Velde also concurred, vocalising that long-term follow-up targeting biomarkers offers a dynamic solution to provision of snapshot information. She further explained: "I really want to emphasise the additional advantage that is, if you have longitudinal time points, you can do repeated measurements, and you can do animal studies with much fewer animals and still have high statistical power in your experiments." In this way, the speakers emphasised that longitudinal input would help provide a baseline for using imaging technology for evaluating lung disease progression and therapy.

\section{CONCLUDING REMARKS}

The information delivered at this ERS session by clinicians at the forefront of the field provides an update to the analysis techniques and imaging biomarkers for lung disease. The discussions will guide future studies and hone medical practice, raising awareness for the requirement of a longitudinal approach. 